\title{
Spectrally Bounded Operators on Simple C*-Algebras, II
}

\author{
MARTIN MATHIEU
}

Dedicated to Professor Gerd Wittstock on the Occasion of his Retirement.

\begin{abstract}
A linear mapping $T$ from a Banach algebra into another Banach algebra is called spectrally bounded if there is a constant $M \geq 0$ such that $r(T x) \leq M r(x)$ for all $x$ in the domain, where $r(\cdot)$ denotes the spectral radius. We investigate to what extent a unital spectrally bounded operator from a simple unital $C^{*}$-algebra of real rank zero onto a unital semisimple Banach algebra is a Jordan epimorphism.
\end{abstract}

\section{INTRODUCTION}

Let $A$ and $B$ be unital semisimple Banach algebras over the complex numbers $\mathbb{C}$. An open conjecture of Kaplansky states that every linear surjective mapping $T: A \rightarrow B$ which is spectrum-preserving, that is, $\sigma(T x)=\sigma(x)$ for all $x \in A$ (where $\sigma(x)$ stands for the spectrum of $x$ ), must be a Jordan algebra isomorphism. This conjecture has been confirmed in the case of von Neumann algebras by Aupetit in [2]. An inspection of his proof shows that, in fact, no further assumption is needed on $B$ and that it suffices that the spectral theorem holds in $A$. Thus, an immediate extension of his result is the following.

Theorem 1.1. Let $A$ be a unital $C^{*}$-algebra of real rank zero, and let $B$ be a unital semisimple Banach algebra. Then every surjective spectrum-preserving mapping linear $T: A \rightarrow B$ is a Jordan algebra isomorphism.

2000 Mathematics Subject Classification. 47B48; 46L05, 47A65, 17C65.

Key words and phrases. Spectrally bounded operators, Jordan algebra homomorphisms, simple $C^{*}$-algebras. 
The techniques presently available appear to need the additional hypothesis of real rank zero, see also Lemma 2.1 below, although there is no reason to assume that this restriction should be necessary. Aupetit's result has been generalised to more general classes of mappings such as spectrally bounded ones. In analogy to the notion of a bounded linear operator, we call a linear mapping $T: A \rightarrow B$ spectrally bounded if there is a constant $M \geq 0$ such that $r(T x) \leq M r(x)$ for all $x \in A$; here, and in the following, $r(x)$ denotes the spectral radius of $x$.

Specifically, the following result was obtained in [7].

Theorem 1.2. Let $T: A \rightarrow B$ be a unital surjective spectrally bounded operator from a unital purely infinite simple $C^{*}$-algebra $A$ onto a unital semisimple Banach algebra $B$. Then $T$ is a Jordan epimorphism.

Recall that a simple $C^{*}$-algebra $A$ is said to be purely infinite if every non-zero hereditary $C^{*}$-subalgebra is infinite. In particular, every non-zero projection has to be infinite and $A$ has no non-zero bounded traces. It is well known [14] that every purely infinite simple $C^{*}$-algebra has real rank zero. It was shown in [7, Lemma 3] that, for every projection $p$ in $A$, the elements with square zero in $p A p$ linearly span $p A p$. This, together with a technique developed in [9], see Lemma 2.3 below, allows us to prove Theorem 1.2.

There are, however, other simple $C^{*}$-algebras with real rank zero in which every element is a finite sum of elements with square zero; see, e.g., $[4,5]$. It is thus close at hand to ask to what extent the approach of controlling a spectrally bounded map on square-zero elements can lead to the conclusion of Jordan epimorphism. We will answer this question in the following in the absence of tracial states (Theorem 3.1). Note that there exist unital simple finite $C^{*}$ algebras (even nuclear, separable ones) without tracial states $[12$, Corollary 7.2].

\section{Some Preliminaries}

Let $T: A \rightarrow B$ be a linear mapping. We shall call $T$ a Jordan epimorphism provided $T$ is surjective and it is a Jordan algebra homomorphism, that is,

$$
T(x y+y x)=(T x)(T y)+(T y)(T x) \quad(x, y \in A) .
$$


It is easy to see that the latter property is equivalent to the requirement $T\left(x^{2}\right)=(T x)^{2}$ for all $x \in A$. If, in addition, $T$ is injective, then we call $T$ a Jordan algebra isomorphism (in order to avoid confusion with the well-established terminology Jordan isomorphism in $C^{*}$-algebra theory which assumes $T$ to preserve the involution as well).

It is well known that every Jordan epimorphism is unital, that is, $T 1=1$ and preserves invertible elements. Hence, $T$ is spectrally bounded with $M=1$. On the other hand, every normalised bounded centre-valued trace on a unital $C^{*}$-algebra is spectrally bounded, and every spectrally bounded operator into a commutative $C^{*}$-algebra is a trace [6]. Dually, every bounded operator from a commutative $C^{*}$-algebra is spectrally bounded. These facts appear to give some evidence to the statement that Jordan epimorphisms and traces constitute the essential examples of spectrally bounded operators. Indeed, if $A$ is a unital $C^{*}$-algebra, $\tau$ a tracial state of $A$ (that is, a positive trace functional of norm one), and $S: A \rightarrow B$ a Jordan epimorphism onto a unital semisimple Banach algebra $B$, then

$$
T=\gamma S+(1-\gamma) \tau \quad(\gamma \in \mathbb{C})
$$

defines a unital spectrally bounded operator on $A$. If $\gamma \neq 0$ then $T$ is surjective. Indeed, let $y \in B$ and $z \in A$ such that $S z=y$. Put $x_{1}=\frac{1}{\gamma}(z-\tau(z) 1)$ and $x_{2}=\tau(z) 1$. Then $x_{1} \in \operatorname{ker} \tau$. Setting $x=x_{1}+x_{2}$ we have

$$
\begin{aligned}
T x & =T\left(x_{1}+x_{2}\right)=\gamma S x_{1}+\gamma S x_{2}+(1-\gamma) \tau\left(x_{1}+x_{2}\right) \\
& =S z-\tau(z) 1+\gamma \tau(z) 1+(1-\gamma) \tau(z) 1 \\
& =S z=y .
\end{aligned}
$$

It is conceivable that, if $A$ is simple, every unital surjective spectrally bounded operator on $A$ is of the form (1). No counterexample is known so far, and the statement is true if $\operatorname{dim} A<\infty$ in addition [13]. However, the argument in [13] rests on a result by Botta, Pierce and Watkins, which does not seem to have an equivalent in infinite dimensions.

One of the principal techniques to show that a bounded linear operator is a Jordan algebra homomorphism has been to control its behaviour on projections. The following lemma is by now standard; 
a proof in the case of von Neumann algebras can be found in $[9$, Lemma 2.1] and takes over verbatim, in view of the results in [3].

Lemma 2.1. Let $T: A \rightarrow B$ be a bounded linear operator from a $C^{*}$-algebra $A$ of real rank zero into a Banach algebra $B$ sending projections in $A$ to idempotents in $B$. Then $T$ is a Jordan algebra homomorphism.

Evidently every Jordan algebra homomorphism $T$ preserves elements of square zero, that is, $(T x)^{2}=0$ for every $x$ with $x^{2}=0$. That spectrally bounded operators have this property as well was shown in [9, Lemma 3.1].

Lemma 2.2. Let $T: A \rightarrow B$ be a spectrally bounded operator from a $C^{*}$-algebra $A$ onto a semisimple Banach algebra $B$. Then $T$ preserves square-zero elements.

The link between this property of a spectrally bounded operator stated in Lemma 2.2 and the hypothesis needed to employ Lemma 2.1 is provided by the following technique, which has been used in both [9] and [7].

Lemma 2.3. Let $T: A \rightarrow B$ be a unital linear operator from a unital $C^{*}$-algebra $A$ into a unital Banach algebra $B$ preserving square-zero elements. Suppose that, for every projection $p \in A$, the hereditary $C^{*}$-subalgebra $p A p$ is linearly spanned by square-zero elements in $p A p$. Then $T$ sends every projection in $A$ onto an idempotent in $B$.

Proof. Let $p$ be a non-zero projection in $A$ such that $q=1-p$ is non-zero as well. If $a \in p A p$ and $b \in q A q$, then, by assumption, there are finitely many $a_{i} \in p A p, b_{j} \in q A q$ such that $a=\sum_{i} a_{i}$, $b=\sum_{j} b_{j}$, and $a_{i}^{2}=b_{j}^{2}=0$ for all $i, j$. We claim that

$$
(T a)(T b)+(T b)(T a)=0 .
$$

Since $\left(a_{i}+b_{j}\right)^{2}=0$ for all $i, j$, the hypothesis on $T$ entails that $\left(T\left(a_{i}+b_{j}\right)\right)^{2}=0$ for all $i, j$. On the other hand,

$$
\begin{aligned}
\left(T\left(a_{i}+b_{j}\right)\right)^{2} & =\left(T a_{i}\right)^{2}+\left(T a_{i}\right)\left(T b_{j}\right)+\left(T b_{j}\right)\left(T a_{i}\right)+\left(T b_{j}\right)^{2} \\
& =\left(T a_{i}\right)\left(T b_{j}\right)+\left(T b_{j}\right)\left(T a_{i}\right),
\end{aligned}
$$

wherefore $\left(T a_{i}\right)\left(T b_{j}\right)+\left(T b_{j}\right)\left(T a_{i}\right)=0$ for all $i, j$. Summing over all indices yields the claim $(2)$. 
Applying (1) to $a=p$ and $b=1-p$ yields

$$
2\left(T p-(T p)^{2}\right)=(T p)(1-T p)+(1-T p)(T p)=0,
$$

as $T 1=1$. Consequently, $T p$ is idempotent.

The following result seems to belong to the folklore; a proof can be found, e.g., in [5, Lemma 3.10].

Lemma 2.4. Let $A$ be a simple unital $C^{*}$-algebra without tracial states. For each projection $p$ in $A$, the hereditary $C^{*}$-subalgebra pAp has no tracial states.

\section{The Main Result}

In view of the remarks in Section 2 we have to rule out the existence of tracial states on a unital $C^{*}$-algebra $A$ in order to make it possible that every unital surjective spectrally bounded operator from $A$ is a Jordan epimorphism. This is equivalent to the requirement that the so-called spectral dual $A^{\sigma}$ of $A$ reduces to $\{0\}$ and, more generally, that every spectrally bounded operator from $A$ into a commutative $C^{*}$-algebra vanishes; see [8] and [6, Corollary 2.3]. The main result in [9] states that, for von Neumann algebras, the non-existence of tracial states is also sufficient. Theorem 3.1 below is the analogue of this result for unital simple $C^{*}$-algebras with real rank zero and hence extends Theorem 1.2 above.

Theorem 3.1. Let $T: A \rightarrow B$ be a unital surjective linear mapping from a unital simple $C^{*}$-algebra $A$ with real rank zero and without tracial states onto a unital semisimple Banach algebra $B$. The following conditions are equivalent:

(a) $T$ is spectrally bounded;

(b) $T$ is bounded and preserves square-zero elements;

(c) $T$ is a Jordan epimorphism.

Proof. The implication (c) $\Rightarrow$ (a) was mentioned at the beginning of Section 2, and (a) $\Rightarrow$ (b) follows from [1, Theorem 1] and Lemma 2.2. Hence assume that (b) holds. Let $p$ be a non-trivial projection in $A$. By $[15$, Theorem I], there exist mutually orthogonal, equivalent subprojections $p_{i}, 1 \leq i \leq 2$ of $p$ and a projection $p_{3}$ which is orthogonal to both $p_{i}$ 's and equivalent to a subprojection of $p_{1}$ such that $p=p_{1}+p_{2}+p_{3}$. Since $p_{i}$ is equivalent to a subprojection of $p-p_{i}$ for each $i=1,2,3$, it follows from [4, Theorem 3.5], see also [5, Remarks 
after Theorem 5.2], that every commutator in $p A p$ is the sum of 14 elements in $p A p$ of square zero. By Lemma 2.4, $p A p$ has no tracial states wherefore [11] entails that every element in $p A p$ is a finite sum of commutators. Combining these two facts we find that $p A p$ is linearly spanned by its square-zero elements; thus, $T$ sends projections in $A$ onto idempotents in $B$ (Lemma 2.3). From Lemma 2.1 we now conclude that $T$ is a Jordan algebra homomorphism. This proves (c).

The above result together with its analogue for properly infinite von Neumann algebras obtained in [9, Corollary 3.5 and Theorem 3.6] makes us wonder whether the following might be true.

Conjecture 3.2. The following two conditions on a unital $C^{*}$ algebra $A$ are equivalent:

(a) A has no tracial states;

(b) every unital bounded operator $T$ preserving square-zero elements from $A$ onto a unital semisimple Banach algebra $B$ is a Jordan epimorphism.

Evidently, (b) $\Rightarrow$ (a) so that the open question is whether (a) implies (b). If this holds, then a related open problem would be solved for unital $C^{*}$-algebras without tracial states, see [8]: Every unital spectral isometry, that is, spectral radius preserving operator, onto a unital $C^{*}$-algebra is a Jordan algebra isomorphism. This is known to be true for a large number of von Neumann algebras, see [10], and no example of a $C^{*}$-algebra where it fails has been found.

Theorem 3.1 together with the results in [10] enable us to treat another case.

Corollary 3.3. Let $T: A \rightarrow B$ be a unital spectral isometry from a unital $C^{*}$-algebra $A$ onto the $C^{*}$-algebra $B=C(X) \otimes C$, where $X$ is a totally disconnected compact Hausdorff space and $C$ is a simple unital $C^{*}$-algebra of real rank zero without tracial states. Then $T$ is a Jordan algebra isomorphism.

Proof. Since $T$ is injective [8, Proposition 4.2], the inverse $T^{-1}$ of $T$ is a unital spectral isometry from $B$ onto $A$. Thus, by interchanging the roles of $A$ and $B$, we can put the hypothesis on $A$, which simplifies the presentation of the argument.

Let $I$ be a Glimm ideal of $A$, that is, the closed ideal generated by a maximal ideal in the centre $Z(A)$ of $A$ (which is isomorphic to 
$C(X))$. Then $I$ is of the form $I=\left\{f \in C(X, C) \mid f\left(x_{0}\right)=0\right\}$ for some point $x_{0} \in X$ (where we identify $C(X) \otimes C$ with $C(X, C)$ ), and the quotient $A / I$ is isomorphic to $C$. By [8, Corollary 4.4], $T Z(A)=Z(B)$ and the restriction of $T$ to $Z(A)$ is an isomorphism between $Z(A)$ and $Z(B)$; in particular, it maps maximal ideals in $Z(A)$ onto maximal ideals in $Z(B)$. Moreover, as $X$ is totally disconnected, $C(X)$ has real rank zero, so the spectral theorem holds. The argument used for von Neumann algebras in [10, Corollary 8] thus takes over and [10, Proposition 10] therefore entails that $J=T I$ is a Glimm ideal in $B$ and that the induced mapping $\hat{T}: A / I \rightarrow B / J$ is a unital spectral isometry. From Theorem 3.1 we hence conclude that $\hat{T}$ is a Jordan algebra isomorphism. Since the Glimm ideals (in $A$ and in $B$ ) separate the points, we finally obtain that $T$ itself is a Jordan algebra isomorphism as claimed.

\section{REFERENCES}

[1] B. Aupetit, The uniqueness of the complete norm topology in Banach algebras and Banach Jordan algebras, J. Funct. Anal. 47 (1982), 1-6.

[2] B. Aupetit, Spectrum-preserving linear mappings between Banach algebras or Jordan-Banach algebras, J. London Math. Soc. 62 (2000), 917-924.

[3] L. G. Brown and G. K. Pedersen, $C^{*}$-algebras of real rank zero, J. Funct. Anal. 99 (1991), 131-149.

[4] L. W. Marcoux, On the linear span of the projections in certain simple $C^{*}$-algebras, Indiana Univ. Math. J. 51 (2002), 753-771.

[5] L. W. Marcoux, Sums of small numbers of commutators, preprint 2004.

[6] M. Mathieu, Spectrally bounded traces on $C^{*}$-algebras, Bull. Austral. Math. Soc. 68 (2003), 169-173.

[7] M. Mathieu, Spectrally bounded operators on simple $C^{*}$-algebras, Proc. Amer. Math. Soc. 132 (2004), 443-446.

[8] M. Mathieu And G. J. Schick, First results on spectrally bounded operators, Studia Math. 152 (2002), 187-199.

[9] M. Mathieu And G. J. Schick, Spectrally bounded operators from von Neumann algebras, J. Operator Theory 49 (2003), 285-293.

[10] M. Mathieu And A. R. Sourour, Hereditary properties of spectral isometries, Arch. Math. (Basel) 82 (2004), 222-229.

[11] C. Pop, Finite sums of commutators, Proc. Amer. Math. Soc. 130 (2002), 3039-3041.

[12] M. RøRDAm, A simple $C^{*}$-algebra with a finite and an infinite projection, Acta Math. 191 (2003), 109-142.

[13] P. ŠEmRL, Spectrally bounded linear maps on $B(H)$, Quart. J. Math. Oxford (2) 49 (1998), 87-92.

[14] S. Zhang, A property of purely infinite simple $C^{*}$-algebras, Proc. Amer. Math. Soc. 109 (1990), 717-720. 
[15] S. Zhang, Matricial structure and homotopy type of simple $C^{*}$-algebras with real rank zero, J. Operator Theory 26 (1991), 283-312.

M. Mathieu,

Department of Pure Mathematics,

Queen's University Belfast,

Belfast BT7 1NN

m.m@qub.ac.uk

Received on 2 February 2005. 